\title{
Pyrolysis Mass Spectrometry: A New Method to Differentiate Between the Mycobacteria of the 'Tuberculosis Complex' and Other Mycobacteria
}

\author{
By GERHAN WIETEN, ${ }^{1 *}$ JOHAN HAVERKAMP, ${ }^{1}$ HENK L. C. \\ MEUZELAAR, ${ }^{1} \dagger$ H. W. BOUDEWIJN ENGEL ${ }^{2}$ AND LUCAS G. BERWALD ${ }^{2}$ \\ ${ }^{1}$ F.O.M. Institute for Atomic and Molecular Physics, Kruislaan 407, 1098 SJ Amsterdam, \\ The Netherlands \\ ${ }^{2}$ National Institute of Public Health, Antonie van Leeuwenhoeklaan 1, 3721 MA Bilthoven, \\ The Netherlands
}

(Received 2 April 1980; revised 21 May 1980)

\begin{abstract}
Pyrolysis mass spectrometry (p.m.s.) was applied to differentiate 91 coded mycobacteria as belonging to either the 'tuberculosis complex' (M. tuberculosis, $M$. bovis and M. bovis BCG) or to other species of Mycobacterium. Strains were analysed in batches; in each batch three strains each of $M$. tuberculosis, $M$. bovis and $M$. bovis BCG were included as references. Mass spectra were evaluated by computerized multivariate analysis. A $92 \%$ positive correlation with classical identification tests was found $(2.2 \%$ false negative, $5.5 \%$ false positive). Approaches for further improvement of this score are indicated. High speed and complete automation of sample analysis, as well as computerized data-processing, make p.m.s. a potential tool for routine application. Sample transfer from peripheral laboratories to a p.m.s. facility is easy, as samples can be sterilized before shipping.
\end{abstract}

\section{INTRODUCTION}

Identification of mycobacteria by conventional methods is a laborious and time-consuming procedure. The selection of adequate criteria as well as their evaluation by means of numerical techniques has been described for most clinically important mycobacteria (Kubica et al., 1973; Meissner et al., 1974; Saito et al., 1977; Tsukamura \& Mizuno, 1977; Wayne et al., 1971, 1978). In practice, the choice of criteria may still vary more or less from laboratory to laboratory and automation is seldom used in the final identification. Attempts have been made to design simpler (Kubica, 1973; Marks, 1976; Pattyn \& Portaels, 1972) and less time-consuming and standardized (Wayne et al., 1974, 1976) means of identification, especially for the separation of Mycobacterium tuberculosis and $M$. bovis from other mycobacteria (Coster \& Manten, 1956; Gruft, 1976; Laszlo \& Eidus, 1978; Marks, 1976; Tsukamura, 1970). Provided sufficient actively multiplying bacteria are available, it is possible to identify $M$. tuberculosis within $2 \mathrm{~h}$ (i.e. by the niacin test, etc.). However, for other species, especially $M$. bovis, $10 \mathrm{~d}$ to 3 weeks are needed to get a definitive identification.

Pyrolysis mass spectrometry (p.m.s.) is a universal technique for the characterization of complex biological material (Meuzelaar et al., 1974). The method can be defined as controlled thermal degradation in an inert environment, i.e. the vacuum of the mass spectrometer (Levy, 1966). The reproducible degradation of originally non-volatile organic matter results in the formation of a mixture of largely volatile products (pyrolysate), suitable for analysis by mass spectrometry. The pyrolysis products are highly dependent on the composition of the starting material. Thus, the mass spectra can be used as fingerprints and give characteristic biochemical information on the samples under investigation. P.m.s.

$\dagger$ Present address: Department of Pathology, School of Medicine, University of Utah, Salt Lake City, Utah, U.S.A. 
provides the possibility for automation and computerization of both the analysis and data-processing procedures (Meuzelaar et al., 1976). Handling of the spectra by computer offers the possibility of applying pattern recognition procedures using multivariate analysis techniques for identification as well as for classification (Eshuis et al., 1977; Sneath \& Sokal, 1973).

In a previous paper concerning the application of p.m.s. in microbiology (Meuzelaar et al., 1977), discrimination between a number of mycobacterial species was reported, and the possibility of matching 'unknown' strains with library strains was indicated.

The conventional distinction between the 'tuberculosis complex' ( $M$. tuberculosis, $M$. bovis and $M$. bovis BCG) and other mycobacteria is of clinical relevance. The present paper describes the application of p.m.s., using automated and computerized sample analysis and data-processing procedures, to the identification of the 'tuberculosis complex'. P.m.s. spectra can be used as an independent starting point for classification purposes; however, such a classification based on physico-chemical data does not necessarily correspond with conventional classification based on other criteria. It was for this reason that the data-processing procedure presented in this paper was developed to give a classification that simulated the classical classification.

\section{METHODS}

Bacterial strains. In total, 91 test strains were selected from those received by the National Institute of Public Health (N.I.P.H.) for routine identification. Mycobacterium bovis BCG strains came from a collection of BCG strains used in laboratories throughout the world for preparation of BCG vaccines and were received through the courtesy of Dr E. Mankiewicz (Chest Hospital Center, Montreal, Canada) and Dr H. L. David (Institut Pasteur, Paris, France) (Mankiewicz \& Liivak, 1971; David, 1978).

Microbiological routine identification. The following classical tests were used for differentiating $M$. tuberculosis, $M$. bovis and $M$. bovis BCG from other mycobacteria: stimulation of growth on medium containing pyruvic acid; niacin production; nitrate reduction; urease, nicotinamidase and pyrazinamidase activity; and sensitivity to thiophene-2-carboxylic acid hydrazide $(\mathrm{TCH}, 1 \mu \mathrm{g} \mathrm{ml}-1)$. The identification scheme is presented in Table 1. Mycobacteria not belonging to the 'tuberculosis complex' were identified using standard methods (Kubica, 1973; Pattyn \& Portaels, 1972).

Sample preparation for p.m.s. analysis. For each strain, streak cultures were made in duplicate on standard Löwenstein medium and incubated at $37^{\circ} \mathrm{C}$ for 3 weeks. Then a small amount of growth was scraped off and emulsified in a drop of sterile, distilled water on a clean glass slide (the glass slide was cleaned for 10 min in an alkali/methanol solution). A ferromagnetic wire was turned slowly in the suspension; in this way, sufficiently reproducible amounts of bacterial mass (about $10 \mu \mathrm{g}$ organic material) remained attached to the wires, as judged from total signal intensities. Duplicate samples were prepared from each suspension. Thus, taking into account the duplicate cultures, four samples were obtained for each strain. Each wire was then mounted in a glass sample holder (Meuzelaar et al., 1975) and sterilized in a pressure cooker for $30 \mathrm{~min}$ at $120^{\circ} \mathrm{C}$. After coding, the samples were stored at $4{ }^{\circ} \mathrm{C}$ and transferred from the N.I.P.H. to the F.O.M. Institute for p.m.s. analysis the next day.

Pyrolysis mass spectrometry. The p.m.s. apparatus used has been described in detail elsewhere (Meuzelaar et al., 1976). Briefly, it consists of a fully automated system with sample changing device, capable of handling 30 samples per hour. Pyrolysis is accomplished by high frequency inductive heating of the ferromagnetic wire to the Curie temperature within $0.1 \mathrm{~s}$. In this study $\mathrm{Fe} / \mathrm{Ni}$ wires with Curie temperatures of $510^{\circ} \mathrm{C}$ were used. The volatile products in the mixture of components were ionized by low energy $(15 \mathrm{eV})$ electron impact. The mass range of the quadrupole mass spectrometer was 15 to 160 a.m.u. The pyrolysate was available for mass spectrometric analysis during approximately $10 \mathrm{~s}$. Rapid multiple scanning of the mass spectrum and accumulation of the signal with a minicomputer in a signal averaging mode were used, and spectra were transferred to a large time-shared computer (C.D.C. 7600) for further data-processing.

Data-processing. The first step in data-processing consisted of a normalization procedure to correct for variations in sample size. Peak intensities were expressed as percentages of the total ion current. Further data-processing was carried out by multivariate analysis techniques described by Eshuis et al. (1977). Every peak of the mass spectra was weighted by the ratio of specificity and reproducibility. Reproducibility can be estimated by calculating intra-strain deviations and includes variation due to culturing as well as sample preparation and analysis. Specificity can be estimated by calculating inter-strain deviations. This inter- to intra-strain ratio, the 'characteristicity' value, is comparable to a Fisher ratio and, to some extent, to a signal to noise ratio. After this procedure only masses with characteristicity values exceeding 1.00 were used for numerical comparison of the 
Table 1. Microbiological tests used for the identification of M. tuberculosis, $M$. bovis and $M$. bovis $B C G$

Feature

Growth rate

Pigment in dark

Pigment, photoactive

Growth stimulation by pyruvic acid

Niacin production

Nitrate reduction

Urease

Nicotinamidase

Pyrazinamidase

Sensitivity to thiophene-2-

carboxylic acid hydrazide

(1 $\mu \mathrm{g} \mathrm{ml}^{-1}$ )
M. tuberculosis M. bovis

slow

slow

$-$

$-$

$+$

$+$

$+$

$+$

$+$
M. bovis $\mathrm{BCG}$

slow
-
-
+
-
-
+
-
-
+

slow

$-$

$-$

$\rightarrow$

$\mathrm{v}$

$\mathrm{v}$

$+$

$-$

spectra. Routinely, the 40 mass peaks with highest characteristicity values were used to calculate distance matrices, in which dissimilarities between the spectral patterns are represented by distance values. These distance values were calculated using the modified Euclidean distance formula (Eshuis et al., 1977)

$$
d(X, Y)=\sqrt{\frac{1}{\sum w_{1}} \sum_{i=1}^{40} w_{i}\left(\frac{x_{1}-y_{1}}{\sigma_{1}}\right)^{2}}
$$

in which $X\left(x_{1}, x_{2}, \ldots, x_{40}\right)$ and $Y\left(y_{1}, y_{2}, \ldots, y_{40}\right)$ are two spectra, $x_{1}$ and $y_{1}$ are the intensity values of the 40 peaks with highest characteristicity $w_{1}$ (which is a weight factor for peak $\mathrm{i}$ ), $\sigma_{1}$ is the averaged standard deviation of peak intensity $\mathrm{i}$, and $d$ is the distance between the spectra.

The distance matrix provides the most complete information about the numerical correlations between the various spectra. However, because of the large number of distance values involved it is not always easy to form a mental picture of the overall relationships. Therefore, a visual representation of the distance matrix (data points in 40-dimensional feature space) is made in the form of a two-dimensional, non-linear map. Kruskals non-linear mapping technique (Kruskal, 1964) provides the basis for this procedure. It should be noted that because of distortion produced by the non-linear mapping procedure (as indicated by the stress value), such a map is primarily used to obtain qualitative impressions about clustering tendencies. To make quantitative decisions the distance matrix values should be used.

\section{RESULTS}

Typical p.m.s. spectra of M. tuberculosis, M. bovis BCG and M. bovis are given in Fig. 1. As can be seen from this figure, the differences between the 'pyrograms', indicated by arrows, are relatively small and complex. This complicates evaluation of spectra by visual inspection, especially if large series of spectra have to be analysed. For this reason, computer-assisted comparison of the spectra was used, as outlined in Methods.

As a first step, the intra-species heterogeneity of the three species was investigated, using 35 strains of $M$. tuberculosis, 26 strains of $M$. bovis BCG and 30 strains of $M$. bovis. The largest heterogeneity was found within $M$. bovis, which appeared to form a long drawn-out cluster, whereas $M$. tuberculosis and $M$. bovis BCG showed a far more homogeneous clustering. It is noteworthy that according to p.m.s. analysis $M$. bovis BCG appears to be biochemically closer to $M$. tuberculosis than to $M$. bovis (see Fig. 2). On the basis of these results representative sets of three reference strains for each of the three species were selected. For each batch of unknown strains to be identified the reference strains were cultured simultaneously with the unknown strains and treated in a similar manner. In this way possible effects of imperfect long-term reproducibility, e.g. small variations in culturing, sample preparation and analysis conditions (Windig et al., 1979), were kept to a minimum.

To obtain a preliminary assessment of the possibility of using p.m.s. to differentiate mycobacteria of the 'tuberculosis complex' from other mycobacteria, 17 coded strains were 


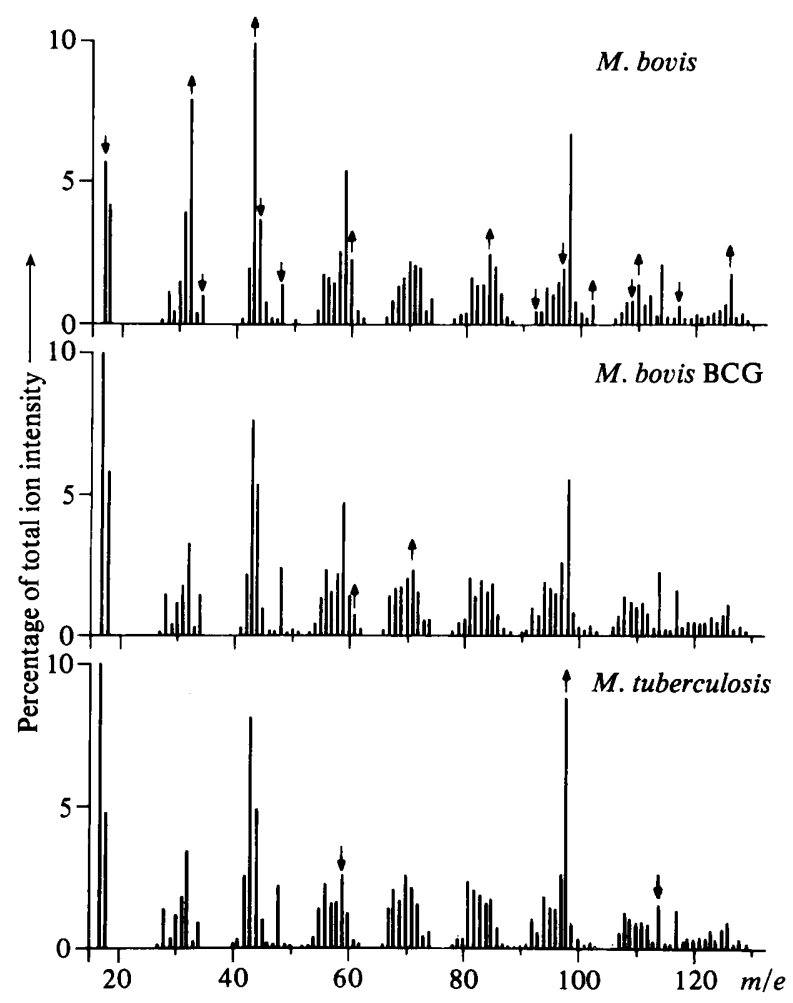

Fig. 1. Typical pyrolysis mass spectra of $M$. bovis, M. bovis BCG and $M$. tuberculosis (mass range of $m / e 130$ to 160 is not shown). Differences between the spectra are indicated by arrows. Note that there are only a few differences between the spectra for $M$. tuberculosis and $M$. bovis BCG, whereas the $M$. bovis spectrum shows many differences from those for $M$. tuberculosis and $M$. bovis BCG.

analysed. The results of multivariate analysis of the spectra are summarized in Table 2 , in the form of an ordered distance matrix. A value on the diagonal represents the average distance between the four analyses of one strain and is indicative of the reproducibility of these analyses. Each off-diagonal value represents the average of the individual distances between the four analyses of one strain and those of the other strain that is being compared and is a measure of the differences between the two strains. The unknown strains were identified on the basis of these distance values using the following procedure. For each reference species a decisive class distance was calculated and used to classify an unknown strain as belonging to one of these classes, e.g. the 'tuberculosis complex'. The class distance values were determined by two factors. Firstly, for each reference species the average distance between the reference strains was calculated. For $M$. tuberculosis and $M$. bovis BCG the distances between all three strains were used, whereas for $M$. bovis only two distances, i.e. between the central and outwardly situated strains, were used in order to correct for the long drawn-out $M$. bovis cluster. For the data presented in Table 2 the average distance for $M$. tuberculosis was 13 , that for $M$. bovis BCG was 17 and that for $M$. bovis was 25 . These distance values accounted for the larger part of species heterogeneities. Secondly, because of the limited number of reference strains per species these distance values did not account completely for total heterogeneities. Empirically chosen multiplication factors were therefore used to correct for this shortage.

In summary, each class distance value was made up of the average distance between the specific reference strains multiplied by a specific empirical factor. For the data presented in 
Table 2. Distance matrix for a batch of 17 character-coded mycobacteria and reference strains for M. tuberculosis, M. bovis BCG and M. bovis

\begin{abstract}
Reference strains are coded by characters A to I. The matrix was ordered to correspond with the outcome of classical microbiological tests. Diagonal values are the average of the distances between the four analyses of one strain. Off-diagonal values represent the average of individual distances between the analyses of two strains. Decisive class distance values were: $M$. tuberculosis, 20; $M$. bovis BCG, 21; M. bovis, 28.
\end{abstract}

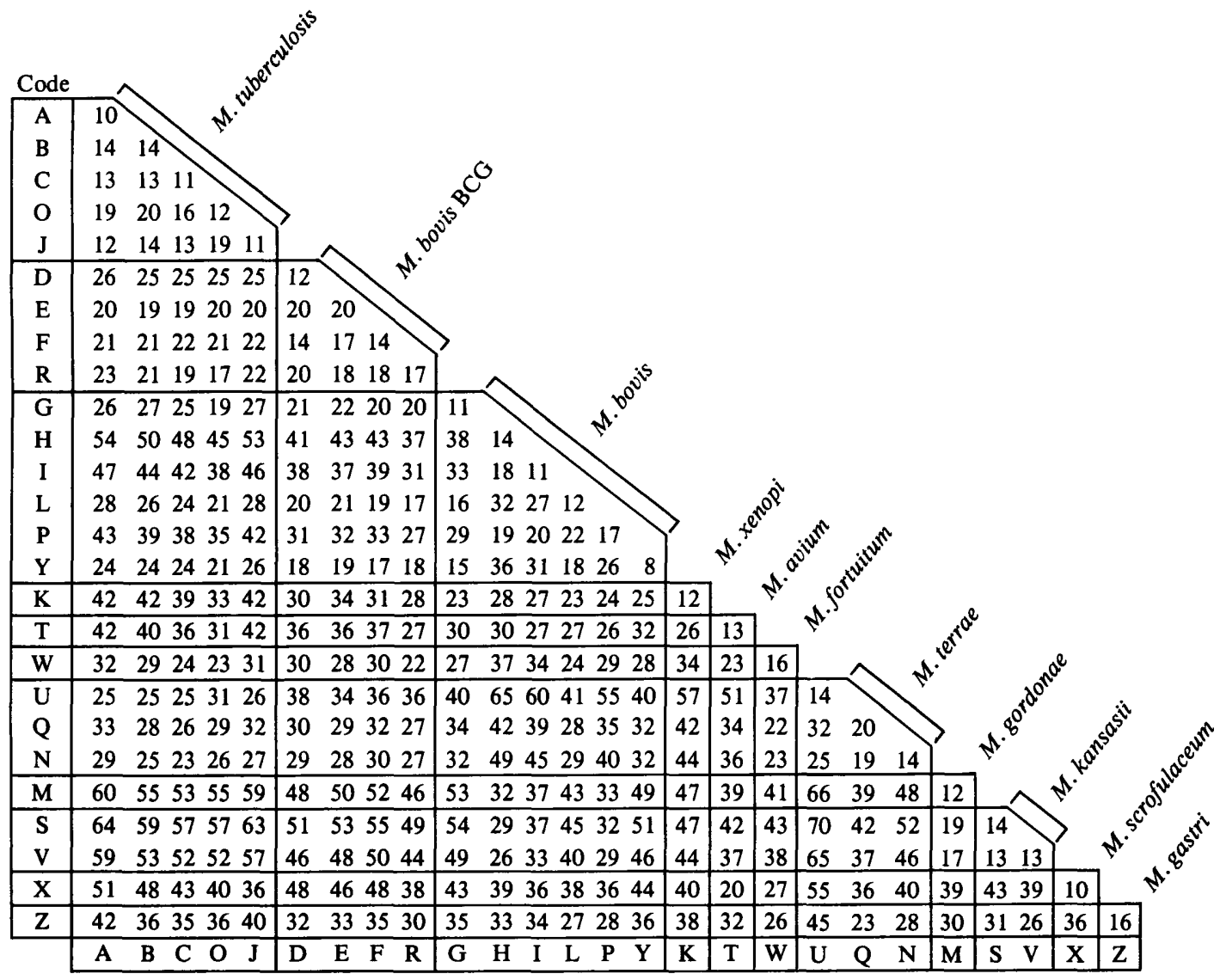

Table 2 the resulting class distance value for $M$. tuberculosis was 20, that for $M$. bovis BCG was 21 and that for $M$. bovis was 28 . Simple comparison of the distance values between an unknown strain and each of the reference strains and the class distance values led to the identification as 'tuberculosis complex' or non-'tuberculosis complex', provided that an unknown strain was within the class distance of a specific reference species for at least two reference strains. It can be concluded from Table 2 that strains $R, K, L, O, P, J$ and $Y$ are closely related to the reference strains. Strains $\mathrm{O}$ and $\mathrm{J}$ were classified as $M$. tuberculosis, strains $\mathrm{Y}$ and $\mathrm{R}$ as $M$. bovis BCG and strains $\mathrm{K}, \mathrm{L}$ and $\mathrm{P}$ as $M$. bovis. The p.m.s. identification agreed well with the classical identification, except that strain $\mathrm{Y}$ was classically identified as $M$. bovis instead of $M$. bovis BCG and strain K was falsely interpreted as $M$. bovis (according to the microbiological identification it was a strain of $M$.xenopi).

A visual representation of the data presented in Table 2 is shown in Fig. 2. From this non-linear map the clustered position of the 'tuberculosis complex' is clearly seen, as well as the relative position of the other species not belonging to this group. The close relationship between $M$. tuberculosis and $M$. bovis BCG and the relatively long drawn-out $M$. bovis 


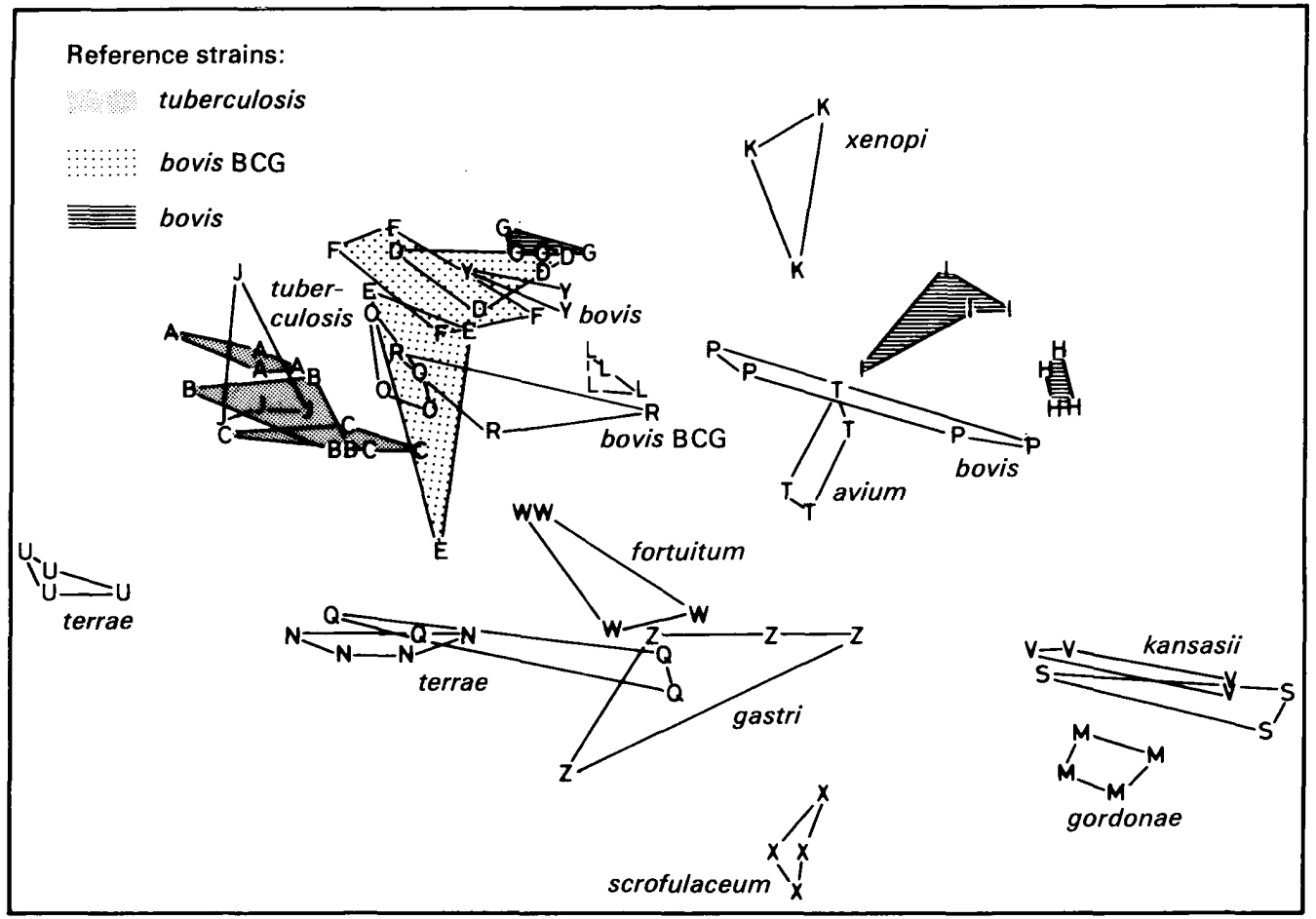

Fig. 2. Non-linear map of the data presented in Table 2. Characters correspond to analyses of strains coded as in Table 2. Each character corresponds to one analysis (spectrum), and analyses of the same strain are connected. Reference strains are shaded. The stress factor (11.3) is indicative of the reliability of the mapping procedure. Note the relatively strong heterogeneity of the $M$. bovis cluster; $M$. tuberculosis and $M$. bovis BCG are more homogeneous and close together.

cluster are well illustrated. Finally, the position of the $M$. avium $(\mathrm{T})$ and $M$. xenopi $(\mathrm{K})$ strains close to the 'tuberculosis complex' should be noted. On the basis of the distance matrix (Table 2) the $M$. avium strain was correctly differentiated from the 'tuberculosis complex', whereas the $M$. xenopi strain was misinterpreted as $M$. bovis. This may illustrate the impossibility of producing a perfect two-dimensional representation of the complex (multidimensional) relationships.

For further evaluation of the applicability of the p.m.s. method, the number of strains to be identified was extended. Results of the p.m.s. analysis of 91 coded strains are summarized in Table 3, together with the results of the classical identification. The classical microbiological identification showed that 10 different species were represented amongst the 58 strains not belonging to the 'tuberculosis complex': $M$. kansasii (10), M. terrae (11), M. avium (7), $M$. gordonae (8), M. gordonae var. ureolyticum (1), M. fortuitum (8), M. xenopi (4), M. gastri (2), $M$. scrofulaceum (2), M. marinum (1) and rapidly growing mycobacteria (4). Using the classical identification as a reference, a positive score of $92 \%$ was found. Of all strains analysed, $2 \cdot 2 \%$ were false negatives and $5.5 \%$ were false positives according to p.m.s. identification.

The false positive strains belonged to the species $M$. xenopi (3), M. avium (1) and to the rapidly growing species, $M$. chitae (1). The strains for which disagreement was found were re-analysed by classical methods. In all cases their identification confirmed the previous classical identification. Re-analysis of one of the $M$. xenopi strains by p.m.s. again resulted in a false positive identification as $M$. bovis. 
Table 3. Comparison between pyrolysis mass spectrometry analysis and microbiological identification tests for 91 mycobacteria Species not belonging to the 'tuberculosis complex' are denoted as 'others'. The microbiological
identification was used as the reference.

\begin{tabular}{|c|c|c|c|c|c|}
\hline & \multicolumn{4}{|c|}{ Pyrolysis mass spectrometry } \\
\hline & & M. tuberculosis & M. bovis $\mathrm{BCG}$ & M. bovis & Others \\
\hline \multirow{4}{*}{$\begin{array}{l}\text { Microbiological } \\
\text { identification }\end{array}$} & M.tuberculosis & 14 & 1 & - & - \\
\hline & M. bovis BCG & 1 & 4 & 一 & 1 \\
\hline & M. bovis & - & 3 & 8 & 1 \\
\hline & Others & 1 & - & 4 & 53 \\
\hline
\end{tabular}

One false negative strain (the Japanese BCG strain) was classically identified as $M$. bovis BCG, whereas the other false negative strain was classically identified as $M$. bovis. Re-analysis of the Japanese $M$. bovis BCG strain by p.m.s. resulted in a correct identification as $M$. bovis BCG. The misinterpreted $M$. bovis strain was not re-analysed by p.m.s. Re-analysis of the false negative strains by classical methods again confirmed the previous microbiological identification.

\section{DISCUSSION}

During pyrolysis, splitting of large molecules into a mixture of smaller fragments occurs. The composition of this mixture is highly dependent on the structure and relative amounts of the original molecules.

Ten of the most reliable peaks for discriminating the 'tuberculosis complex' from other mycobacteria, as shown by consistently high characteristicity values, are the peaks at $m / e 17$, $31,32,48,50,58,59,71,86$ and 98 . On the basis of previous p.m.s. studies ranging from simple reference substances to complex biopolymers and whole micro-organisms (Haider et al., 1977; Posthumus et al., 1974; Schulten et al., 1973; Schulten \& Görtz, 1978; Simmonds, 1970; Weyman, 1977), these ion signals can be tentatively assigned to protein $(\mathrm{m} / \mathrm{e} \mathrm{48})$, carbohydrate $(m / e 86,98)$ and phospholipid $(m / e ~ 50,59,71)$ fragments, whereas the signals at $m / e 17,31,32$ and 58 may have had multiple origins. Obviously, not a single substance or class of substances is responsible for the differences observed, but complex biochemical differences exist between strains of the mycobacterial species analysed.

Out of 91 strains analysed, 84 were correctly classified with respect to their relationship to the 'tuberculosis complex'. Mycobacterium bovis was involved in one way or another in most of the false identifications. From the heterogeneity study, as well as from previous work by Meuzelaar et al. (1977), it is known that $M$. bovis is a very heterogeneous species. The three reference strains for $M$. bovis, although carefully selected on the basis of the heterogeneity study, were clearly not sufficient to characterize this species adequately.

Three of the false positive scores were caused by two different $M$. xenopi strains. In all, this species was analysed four times and it tended to be difficult to differentiate it from the 'tuberculosis complex', in particular from $M$. bovis. The addition of an extra M. bovis reference strain may facilitate the identification of $M$. xenopi strains. In the same way the mis-identification of the $M$. avium strain might have been prevented. The false positive rapidly growing $M$. chitae strain does not pose a serious problem, because rapidly growing mycobacteria are easily detected during the culturing period.

The two false negative strains appeared in the respective distance matrices very close to the class distance values. The false negative Japanese $M$. bovis BCG strain analysed as a coded strain had also been analysed in the earlier heterogeneity study of $M$. bovis BCG and appeared in that study at the border of the cluster. The false negative $M$. bovis strain was not 
re-analysed as a coded strain, neither had it been involved in the heterogeneity study of $M$. bovis.

Calculation of differences between pairs of spectra provided the basic data for identification. Class distances for each of the three reference species were calculated and the maximum overall difference that could be expected to exist between a reference strain and other strains belonging to the same species was estimated. It was found that heterogeneities of $M$. tuberculosis, $M$. bovis BCG and $M$. bovis were not equivalent and thus different class distances were used for each of the three species.

In the present study the 40 features with highest characteristicity values were routinely used for the calculation of distance matrices. All these features have ratios exceeding 1.00 and thus contribute information. However, some of these features may carry non-relevant information ('noise') concerning differences between strains not belonging to the 'tuberculosis complex', rather than information relevant to the differentiation of the 'tuberculosis complex' from other mycobacteria. Consequently, either a reduction in the number of features used for calculation or the selection of 'key features' is necessary (Wieten et al., 1980) and may lead to a more sensitive and reliable differentiation of the 'tuberculosis complex'. Preliminary studies showed that reduction of the number of features from 40 to 10 resulted in an improvement of the characteristicity of the distance matrix (Eshuis et al., 1977) and apparently in a reduction of the number of mis-identified strains.

The use of a single set of 'key features' requires good long-term reproducibility. The data discussed in this paper were collected over a period of approximately 1 year. During this period the apparatus was dismantled for cleaning and maintenance, but still the outcome of feature selection procedures of the various series of analyses showed a fair degree of correspondence. A set of seven 'key features' (namely, $m / e$ 31, 50, 58, 59, 71, 86 and 98) has been selected and will be used for further studies.

In conclusion, it can be stated that p.m.s. procedures show excellent promise for routine investigations of mycobacteria. At present the correspondence with the results of conventional classification may still fall somewhat short for reliable routine application. However, there are strong indications that data-processing can be improved, resulting in a reduction of the number of false negative as well as false positive strains. Furthermore, adaption of the set of reference strains may also improve the score.

The main features of the p.m.s. system are speed of analysis together with computerization up to the level of identification. Further results (G. Wieten and others, unpublished) reveal that the sterilization procedure used neither introduces any loss of reproducibility nor lowers the identification score. Therefore pathogenic samples can easily be transferred from the culturing laboratory to a central facility for p.m.s. analysis.

Finally, as shown by previous work (Eshuis et al., 1977; Meuzelaar et al., 1975; Weyman, 1977), the use of p.m.s. is by no means restricted to the classification of mycobacteria; the technique can be applied without basic changes in many other fields of microbiology.

The authors are greatly indebted to J. G. Baas for preparing the samples, to Mrs A. Tom and Mrs B. Brandt for performing the pyrolysis mass spectrometric analysis and to $\mathrm{Dr} \mathrm{W}$. Eshuis for development of the computer program.

This investigation was supported by the Foundation for Fundamental Research on Matter (F.O.M.) and the Ministry of Health and Environmental Hygiene in the Netherlands.

\section{REFERENCES}

Coster, J. F. \& Manten, A. (1956). A simple technique for the differentiation of tubercle bacilli and saprophytic mycobacteria. American Review of Tuberculosis and Pulmonary Diseases 74, 958-960.
DAVID, H. L. (1978). Phenotypes of BCG-vaccines seed lot strains: results of an international cooperative study. Tubercle 59, 139-142.

Eshuis, W., Kistemaker, P. G. \& MeuzelaAR, H. L. 
C. (1977). Some numerical aspects of reproducibility and specificity. In Analytical Pyrolysis, pp. 151166. Edited by C. E. R. Jones \& C. G. A. Cramers. Amsterdam: Elsevier.

GRUFT, H. (1976). Three simple tests as an adjunct to the niacin test for the small mycobacteriology laboratory. Health Laboratory Science 13, 179183.

Haider, K., Nagar, B. R., Saiz, C., Meuzelaar, H. L. C. \& Martin, J. P. (1977). Studies on soil humic compounds, fungal melanins and model polymers by pyrolysis mass spectrometry. In Soil Organic Matter Studies, vol. II, pp. 213-220. Vienna: International Atomic Energy Agency.

KRUSKAL, J. B. (1964). Multidimensional scaling by optimizing goodness of fit to a nonmetric hypothesis. Psychometrica 29, 1-29.

KUBICA, G. P. (1973). Differential identification of mycobacteria. VII. Key features for identification of clinically significant mycobacteria. American Review of Respiratory Disease 107, 9-21.

Kubica, G. P., Silcox, V. A. \& Hall, E. (1973). Numerical taxonomy of selected slowly growing mycobacteria. Journal of General Microbiology 74, 159-167.

LAszlo, A. \& Eidus, L. (1978). Test for differentiation of $M$. tuberculosis and $M$. bovis from other mycobacteria. Canadian Journal of Microbiology 24, 754-756.

LEVY, R. L. (1966). Pyrolysis gas chromatography, review of the technique. Chromatographic Reviews 8, 48-89.

Mankiewicz, E. M. \& LirvaK, M. (1971). Phage types of Mycobacterium bovis substrains of B.C.G. Canadian Journal of Microbiology 23, 818-823.

MARKS, J. (1976). A new practical classification of the mycobacteria. Journal of Medical Microbiology 9, 253-261.

Meissner, G., Schröder, K., Amadio, G. E., Anz, W., Chaparas, S. D., Engel, H. W. B., Jenkins, P. A., Käppler, W., Kleeberg, H. H., Kubala, E., Kubin, M., LaUterbach, D., Lind, A., Magnusson, M., Mikova, Z. D., Pattyn, S. R., Schaefer, W. B., Stanford, J. L., Tsukamura, M., WAYNe, L. G., WILlers, I. \& Wolinsky, E. (1974). A cooperative numerical analysis of nonscoto- and nonphoto-chromogenic slowly growing mycobacteria. Journal of General Microbiology 83, 207-235.

MeuzelaAR, H. L. C., Kistemaker, P. G. \& Posthumus, M. A. (1974). Recent advances in pyrolysis mass spectrometry of complex biological materials. Biomedical Mass Spectrometry 1, 312319.

MeuzelaAR, H. L. C., Kistemaker, P. G. \& Tom, A. (1975). Rapid and automated identification of microorganisms by Curie-point pyrolysis techniques. I. Differentiation of bacterial strains by fully automated Curie-point pyrolysis gas-liquid chromatography. In New Approaches to the Identification of Microorganisms, pp. 165-178. Edited by C.-G. Hedén \& T. Illéni. New York: Wiley.

MeuzelaAR, H. L. C., Kistemaker, P. G., Eshuis, W. \& Boerboom, A. J. H. (1976). Automated pyrolysis mass spectrometry; application to the differentiation of microorganisms. In Advances in
Mass Spectrometry, vol. 7B, pp. 1452-1456. Edited by N. R. Daly. London: Heyden.

Meuzelaar, H. L. C., Kistemaker, P. G., Eshuis, W. \& ENGEL, H. W. B. (1977). Progress in automated and computerized characterization of microorganisms by pyrolysis mass spectrometry. In Rapid Methods and Automation in Microbiology, pp. 225-230. Edited by S. W. B. Newson \& H. H. Johnston. Oxford: Learned Information.

Pattyn S. R. \& Portaels, F. (1972). Identification and clinical significance of mycobacteria. Zentralblatt für Bakteriologie, Parasitenkunde, Infektionskrankheiten und Hygiene (Abteilung I, Originale A) 219, 114-140.

Posthumus, M. A., Boerboom, A. J. H. \& MeuzelaAr, H. L. C. (1974). Analysis of biopolymers by Curie-point pyrolysis in direct combination with low voltage electron impact ionization mass spectrometry. In Advances in Mass Spectrometry, vol. 6, pp. 397-402. Edited by A. R. West. London: Heyden.

SAIto, H., Gordon, R. E., Jublin, I., KÄPPleR, W., Kwapinksi, J. B. G., McDurmont, C., Pattyn, S. R., Runyon, E. H., Stanford, J. L., TARnok, I., TASAKA, H., TsukamuRa, M. \& WeiszFEILER, J. (1977). Cooperative numerical analysis of rapidly growing mycobacteria. International Journal of Systematic Bacteriology 27, 75-85.

Schulten, H.-R. \& GöRTZ, W. (1978). Curie-point pyrolysis and field ionization mass spectrometry of polysaccharides. Analytical Chemistry 50, 428-433.

SCHulten, H.-R., BeCKey, H. D., MeuzelaAR, H. L. C. \& Boerboom, A. J. H. (1973). High resolution field ionization mass spectrometry of bacterial pyrolysis products. Analytical Chemistry 45, 191195.

Simmonds, P. G. (1970). Whole microorganisms studied by pyrolysis gas chromatography-mass spectrometry: significance for extraterrestrial life detection experiments. Applied Microbiology 20, 567-572.

SNEATh, P. H. A. \& Sokal, R. R. (1973). Numerical Taxonomy. San Fransisco: Freeman \& Co.

TsUKAMURA, M. (1970). Screening for atypical mycobacteria. Tubercle 51, 280-284.

Tsukamura, M. \& Mizuno, S. (1977). Numerical analysis of relationships among rapidly growing scotochromogenic mycobacteria. Journal of General Microbiology 98, 511-517.

Wayne, L. G., Dietz, T. M., Gernez-RieuX, C., Jenkins, P. A., KÄPPleR, W., KubicA, G. P., Kwapinsky, J. B. G., Meissner, G., Pattyn, S. P., Runyon, E. H., Schröder, K. H., Silcox, V. A., TACQueT, A., Tsukamura, M. \& Wolinsky, E. (1971). A cooperative numerical analysis of scotochromogenic slowly growing mycobacteria. Journal of General Microbiology 66, 255-271.

WAYNe, L. G., ENGbaEK, H. C., ENGel, H. W. B., Froman, S., Gross, W., Hawkins, J., Käppler, W., Karlson, A. G., KleEberg, H. H., Krasnow, I., Kubica, G. P., McDurmont, C., Nel, E. E., PatTyn, S. R., Schröder, K. H., Showalter, S., TARnOK, I., Tsukamura, M., VERgmanN, B. \& WolINSKY, E. (1974). Highly reproducible techniques for use in systematic bacteriology in the genus Mycobacterium: tests for pigment, urease, 
resistance to sodium chloride, hydrolysis of Tween 80 and $\beta$-galactosidase. International Journal of Systematic Bacteriology 24, 412-419.

Wayne, L. G., Engel, H. W. B., Grassi, C., Gross, W., Hawkins, J., Jenkins, P. A., KÄPpleR, W. Kleeberg, H. H., Krasnov, I., Nel, E. E., Pattyn, S. R., Richards, P. A., Showalter, S., Slosarek, M., Szabo, I., Tarnok, I., TSUKamura, M., VergmanN, B. \& Wolinsky, E. (1976). Highly reproducible techniques for use in systematic bacteriology in the genus Mycobacterium: tests for niacin and catalase and for resistance to ioniazid, thiophene 2-carboxylic acid hydrazide, hydroxylamine and $p$-nitrobenzoate. International Journal of Systematic Bacteriology 26, 311-318.

Wayne, L. G., ANdrade, L., Froman, S., Käppler, W., Kubala, E., Meissner, G. \& Tsukamura, M. (1978). A cooperative numerical analysis of Myco- bacterium gastri, Mycobacterium kansasii and Mycobacterium marinum. Journal of General Microbiology 109, 319-327.

WEYMaN, A. C. M. (1977). The application of Curie-point pyrolysis mass spectrometry in fungal taxonomy. In Analytical Pyrolysis, pp. 225-233. Edited by C. E. R. Jones \& C. A. Cramers. Amsterdam: Elsevier.

Wieten, G., Haverkamp, J., Engel, H. W. B. \& BERWALD, L. G. (1980). Application of pyrolysis mass spectrometry in mycobacterial classification and identification. Reviews of Infectious Diseases 2, (in the Press).

Windig, W., Kistemaker, P. C., Haverkamp, J. \& MeuzelaAR, H. L. C. (1979). The effects of sample preparation, pyrolysis and pyrolyzate transfer conditions on pyrolysis mass spectra. Journal of Analytical and Applied Pyrolysis 1, 39-52. 